\title{
Mutation Analysis of the GLUT2 Gene in Patients with Fanconi-Bickel Syndrome
}

\author{
OSAMU SAKAMOTO, EISHIN OGAWA, TOSHIHIRO OHURA, YUTAKA IGARASHI, \\ YOICHI MATSUBARA, KUNIAKI NARISAWA, AND KAZUIE IINUMA
}

Department of Pediatrics [O.S., E.O., T.O., K.I.] and Department of Medical Genetics [Y.M., K.N.], Tohoku University School of Medicine, Sendai, Japan; Igarashi Pediatric Clinic, Sendai, Japan [Y.I.]

\begin{abstract}
Fanconi-Bickel syndrome (FBS) is an autosomal recessive disorder manifesting hepatorenal glycogen accumulation, Fanconi nephropathy, and impaired utilization of glucose and galactose. Several mutations in a gene encoding a glucose transporter, GLUT2, have recently been reported in patients with FBS. We performed molecular analysis on three Japanese patients and found four novel mutations: a splice-site mutation (IVS2-2A>G), a nonsense mutation (Q287X), and two missense mutations (L389P and V423E). Heterozygotes of L389P or V423E mutation from the patients' families showed renal glucosuria. These
\end{abstract}

\section{ABSTRACT}

data suggested that GLUT2 gene defects may be a cause of renal glucosuria. (Pediatr Res 48: 586-589, 2000)
Abbreviations
FBS, Fanconi-Bickel syndrome
GLUT, facilitative glucose transporter
AST, aspartate aminotransferase
ALT, alanine aminotransferase
OGTT, oral glucose tolerance test

FBS (McKusick 227810) is an autosomal recessive disorder of carbohydrate metabolism characterized by hepatorenal glycogen accumulation, Fanconi nephropathy, and impaired utilization of glucose and galactose (1-3). The underlying defect was first identified by Santer et al. (4). They singled out the gene for GLUT2, a facilitative glucose transporter, as a possible site for the basic defect, and they reported three mutations in the GLUT2 gene in three families with FBS. GLUT2 is present in the plasma membrane of pancreatic $\beta$ cells and hepatocytes, as well as absorptive epithelial cells in the intestine and kidney $(5,6)$. Knockout mice lacking a functioning GLUT2 gene manifested hyperglycemia, hypoinsulinemia, and extreme glucosuria (7).

We found four novel mutations in three Japanese patients with FBS, and two of the affected families manifested renal glucosuria.

\section{METHODS}

Patients. Patient 1 was described elsewhere (8). She was born to unrelated parents and was referred to our hospital at $1 \mathrm{y}$

Received January 17, 2000; accepted June 14, 2000.

Correspondence: Toshihiro Ohura, M.D., Ph.D., Department of Pediatrics, Tohoku University School of Medicine, 1-1 Seiryo-machi, Aoba-ku, Sendai, Japan.

The work was supported mainly by Grants-in-Aid for Scientific Research from the Ministry of Education, Culture and Science of Japan, and grants from the Ministry of Health and Public Welfare of Japan (Comprehensive Research on Aging and Health). of age because of hapatomegaly. Growth retardation, rickets, fasting hypoglycemia, glucosuria, and generalized aminoaciduria were also observed. Serum concentrations of liver aminotransferases were high: AST, 109 IU/L; ALT, 62 IU/L. Histologic analysis of biopsied specimens revealed findings compatible with glycogen storage disease. The administration of uncooked cornstarch was started at the age of $5 \mathrm{y}$ and marked catch-up growth was observed.

The brother of this patient was found to have glucosuria on a preemployment check up. The mother also sometimes showed postprandial glucosuria. The father had not been found to have glucosuria at periodic physical examinations.

Patient 2 presented with failure-to-thrive and was diagnosed with Fanconi syndrome at the age of 5 mo. Symptomatic treatment with electrolyte replacement and vitamin D was promptly initiated. Mental development was retarded in addition to growth. Open reductions of femoral fractures were performed at the ages of 3 and $5 \mathrm{y}$. The patient frequently showed fasting hypoglycemia and ketonuria. Hepatomegaly and elevated hepatic transaminases (AST, $110 \mathrm{IU} / \mathrm{L} ; \mathrm{ALT}, 150$ IU/L) were also noted. The patient was admitted to our hospital for evaluation at the age of 15 when he moved into our district. Body height was $125.3 \mathrm{~cm}$ (-7 SD), weight was $23.3 \mathrm{~kg}(-4$ $\mathrm{SD})$, and intelligence quotient Wechsler Intelligence Scale for 
Children-Revised (WISC-R) was 66. The liver edge was palpable $5 \mathrm{~cm}$ below the costal margin. Chemical analyses of urine revealed glucosuria, generalized aminoaciduria, proteinuria, and elevated urine $\beta 2$ microglobulin. This patient's father could not be studied.

Patient 3, born to unrelated healthy parents, was diagnosed with hypergalactosemia by neonatal mass screening at the age of $5 \mathrm{~d}$ (Paigen method, $>1.1 \mathrm{mmol} / \mathrm{L}$; Beutler method, normal). When we first examined the patient at the age of $14 \mathrm{~d}$, hypergalactosemia was confirmed $(2.7 \mathrm{mmol} / \mathrm{L})$. Thin-layer chromatographic analysis of galactose and other metabolites using dried blood spots ruled out galactokinase deficiency and UDP-galactose-4-epimerase deficiency (9). Biochemical analysis revealed intrahepatic cholestasis: total bilirubin, 170 $\mu \mathrm{mol} / \mathrm{L}$ (normal range 3.4-20.4); alkaline phosphatase, 2065 IU/L (normal range 112-330); $\gamma$-glutamyltranspeptidase, 347 IU/L (normal range 8-57); and total bile acids $77.6 \mu \mathrm{mol} / \mathrm{L}$ (normal range 0.7-12.7). Transaminases were within the normal range (AST, $23 \mathrm{IU} / \mathrm{L}$; ALT, $11 \mathrm{IU} / \mathrm{L}$ ). The abnormalities in the blood persisted for $3 \mathrm{mo}$, after which they improved spontaneously. However, the urine Benedict test remained strongly positive even after the serum galactose concentration had been normalized by feeding galactose-free formula. When we measured urine glucose, massive glucosuria $(110 \mathrm{mmol} / \mathrm{d})$ was observed. In addition, generalized aminoaciduria, hypercalciuria, phosphaturia, and elevated urine $\beta 2$ microglobulin were also detected. The patient showed mild fasting hypoglycemia $(3.3 \mathrm{mmol} / \mathrm{L})$ and postprandial hyperglycemia (11.1 $\mathrm{mmol} / \mathrm{L}$ ). Neither hepatomegaly, rickets, nor short stature developed. Glucosuria was not pointed out in either of this patient's parents.

Informed consent was obtained from all of the patients or their families. This study was approved by the Ethical Committee of the Tohoku University School of Medicine.

Direct sequencing of the GLUT2 gene. Genomic DNA was extracted from leukocytes with the aid of a Sepa Gene Kit (Sanko Junyaku, Tokyo, Japan). All 11 exons including flanking introns were amplified by PCR. To facilitate the cycle sequencing reaction, the KS primer sequence (underlined) or the M13 reverse sequence (double-underlined) was attached to the $5^{\prime}$ end of the sense primers (S) or the antisense primers (AS). The sequences of primers for exons 3, 6, 8, and 9 were S-3-KS, 5'-CGAGGTCGACGGTATCGCCAACTGTAAACAAATCTATCC-3'; AS-3Rev, 5'-CAGGAAACAGCTATGACAAAACAACTCTAAAGCTATTCC-3'; S-6-KS, 5'-CGAGGTCGACGGTATCGAAAAAGAATTTCCAACACCCATG-3'; AS-6-Rev, 5' CAGGAAACAGCTATGACAAACTGTACCCCTTGCCTTCCC-3'; S-8-KS, 5'-CGAGGTCGACGGTATCGCTCCCCCACCTTGATCTCACTCC-3'; AS-8-Rev, 5' -CAGGAAACAGCTATGACGTTAATGAAAAACCTCAGTGCAG-3'; and S-9-KS, 5'-CGAGGTCGACGGTATCGGTTGTATGCCGCCTGTGAACTC-3'; AS-9-Rev, 5'-CAGGAAACAGCTATGACGCAGTTTTTTGACCCTCTCTTC-3'. Primer sequences for other exons are available from the authors on request. Direct sequencing of the PCR products was performed using a Thermo Sequenase cycle sequencing kit and an A.L.F. red DNA sequencer (Amersham Pharmacia Biotech, Uppsala, Sweden).
$P C R$ with restriction fragment analyses for IVS2 $-2 A>G$, Q287X, L389P, and V423E mutations. PCR amplification was performed for exons 3,8 , and 9 as described above. PCR products were digested with $A v r I I$ (for IVS2-2A $>\mathrm{G}$ ), BbvI (for L389P), or MnlI (for V423E) (all from New England Biolabs, Beverly, MA, U.S.A.). Fragments were separated on agarose gels (for IVS2-2A>G and L389P, 3\% agarose; for V423E, 6\% agarose) and visualized by ethidium bromide staining. The Q287X mutation was detected by a PCR method incorporating a restriction fragment length polymorphism. The PCR was conducted with a sense primer (S-Q287X: 5'GAGAAGAAGCATCGAGTGCG-3') that contained one mismatch (bold type) to create a $H h a \mathrm{I}$ site, together with antisense primer (AS-6-Rev). After digestion of PCR products with HhaI (New England Biolabs), elecrophoresis was performed using $3 \%$ agarose gel.

Reverse transcription-PCR and subcloning for detection of exon skipping. GLUT2 cDNA was synthesized from total RNA obtained from cultured lymphoblasts with the aid of a Trizol Reagent and a ThermoScript RT-PCR System (GIBCO BRL, Life Technologies, Rockville, MD, U.S.A.). Primers S-1 (5'-ACAAGACCTGGAATTGACAGGACTC-3') and AS-2 (5'-ACGGTTCCCTTATTGTTTCTG-3') were used for the first PCR, and S-1 and AS-1 (5'-TGGTGACATCATCATATCCTCTG-3') were used for the nested second PCR. PCR products were separated on a 3\% agarose gel. Each fragment was purified with a QIAquick Gel Extraction Kit (Promega, Madison, WI, U.S.A.) and inserted into the pT7Blue T-Vector (Novagen, Madison, WI, U.S.A.). Sequences of the inserts were determined as described above.

Oral glucose tolerance test. The OGTT was perfomed by administration of $75 \mathrm{~g}$ of glucose. Samples of blood and urine were obtained for glucose determination before glucose loading and at 1 and $2 \mathrm{~h}$ after loading.

\section{RESULTS}

In patient 1 we found a T-to-A substitution at nt 1580 [nucleotide 1580, numbered from the transcription initiation site (10)] in a homozygous pattern (Fig. 1A), resulting in the replacement of Val with Glu (V423E) within exon 9. PCR products digested with $M n l \mathrm{I}$ also showed that patient 1 was homozygous for the V423E mutation (Fig. 2A). The father, mother, and brother were all heterozygous for this mutation.

In patient 2, direct sequencing of exon 3 and the flanking introns revealed an A-to-G substitution at position -2 of the splice acceptor site (IVS2-2A>G) in a homozygous pattern (Fig. 1B). Reverse transcription and PCR from nt 276 (within exon 1) to nt 1123 (within exon 5) showed aberrant bands of smaller sizes than in the wild-type gene (data not shown). DNA sequencing of subcloned plasmids demonstrated skipping of exon 3, which resulted in a frame shift and created premature termination (data not shown). As shown in Figure $2 B$, the patient was homozygous, whereas his mother was heterozygous for the IVS2 $-2 \mathrm{~A}>\mathrm{G}$ mutation.

In patient 3 we found a C-to-T substitution at nt 1171 in exon 6 and a T-to-C substitution at nt 1478 in exon 8 (Fig. 1, $C$ and $D$ ). The $\mathrm{C} 1171 \mathrm{~T}$ substitution created a stop codon 
A
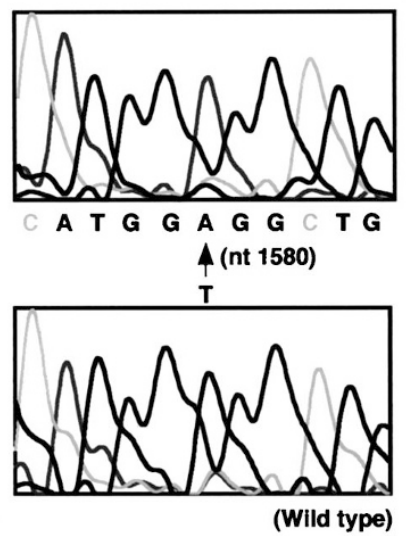

C

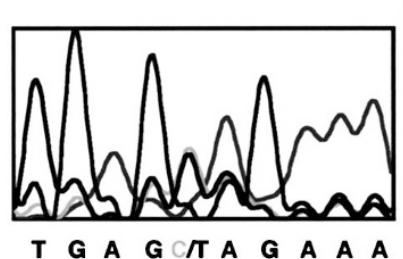
$\Delta(n t 1171)$

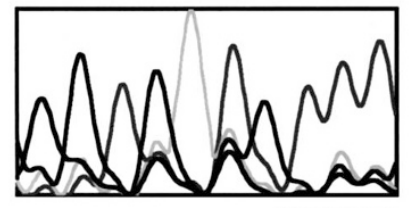

(Wild type)
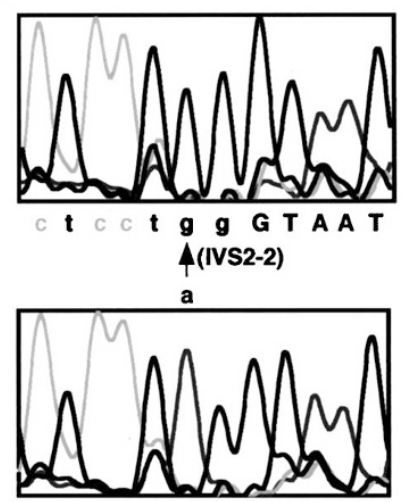

(Wild type)

D
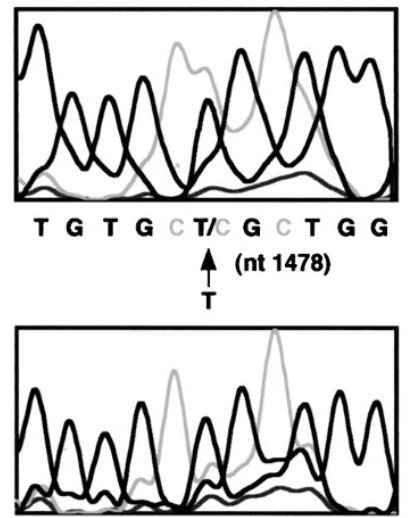

(Wild type)

Figure 1. Results of sequence analysis. Comparisons of the nucleotide sequences of wild-type and mutant alles are shown. (A) Patient 1: A T-to-A substitution at nucleotide (nt) 1580 (V423E) was found in a homozygous pattern. (B) Patient 2: An A-to-G substitution at position -2 of the intron 2 (IVS2-2A $>\mathrm{G}$ ) was found in a homozygous pattern. (C) Patient 3: A C-to-T substitution at nt 1171 (Q287X) was found in a heterozygous pattern. $(D)$ Patient 3: A T-to-C substitution at nt 1478 (L389P) was found in a heterozygous pattern.

(Q287X), and the T1478C resulted in a change from Leu to Pro (L389P). The Q287X mutation was transmitted from the patient's father, and the L389P mutation from the mother (Fig. 2, $C$ and $D$ ).

In a screening for the L389P and V423E mutations in DNA samples from 50 healthy volunteers, neither of the mutations were found in any of the alleles.

OGTT was performed on the family members of patients 1 and 3 (Table 1). The blood glucose profiles of all the family members were normal except that of the mother of patient 1 , who showed a pattern of borderline glucose tolerance impairment. Glucosuria was manifested in the sibling and parents of patient 1 , who were heterozygous for the V423E mutation, and the mother of patient 3, who was heterozygous for the L389P mutation. Urinary glucose was negative in the father of patient 3, a heterozygote of the Q287X mutation.

\section{DISCUSSION}

Fanconi and Bickel were the first investigators to report a patient with a rare autosomal recessive inborn error of metab-

B

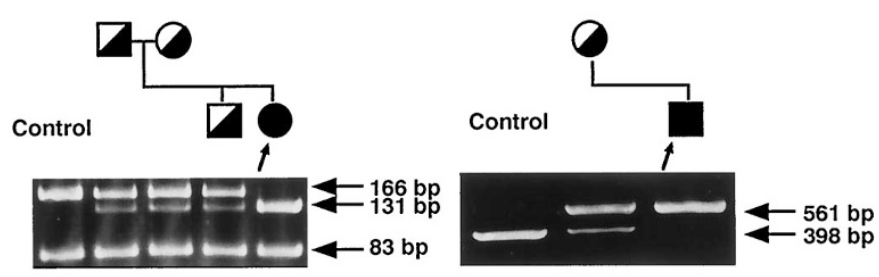

C

D

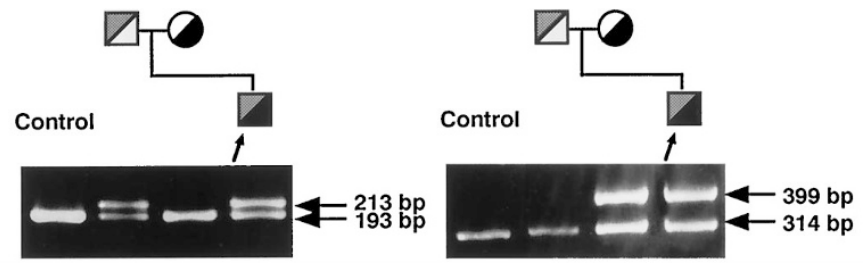

Figure 2. Results of PCR incorporating restriction fragment length polymorphism. (A) V423E creates an additional MnlI site. Patient 1 was homozygous and her father, mother, and brother were heterozygous for V423E. $(B)$ IVS2 $-2 \mathrm{~A}>\mathrm{G}$ abolishes an AvrII site. Patient 2 was homozygous and his mother was heterozygous for IVS2-2A $>$ G. (C) The S-Q287X primer, which includes one mismatch, creates a $H h a \mathrm{I}$ site in the wild-type situation; Q287X abolishes this site. Patient 3 and his father were heterozygous for Q287X. (D) L389P abolishes a $B b v I$ site. Patient 3 and his mother were heterozygous for L389P.

Table 1. Blood and urinary glucose values on OGTT

\begin{tabular}{ccccc}
\hline & & $0 \mathrm{~h}$ & $1 \mathrm{~h}$ & $2 \mathrm{~h}$ \\
\hline Family of patient 1 & & & & \\
Father (Wild/V423E) & $\mathrm{B}$ & 5.6 & 8.8 & 5.0 \\
& $\mathrm{U}$ & 0.5 & 5.6 & 0.7 \\
Mother (Wild/V423E) & $\mathrm{B}$ & 5.6 & 5.7 & 7.9 \\
& $\mathrm{U}$ & 0.5 & 3.7 & 4.2 \\
Brother (Wild/V423E) & $\mathrm{B}$ & 5.4 & 9.2 & 5.2 \\
& $\mathrm{U}$ & $<1.1$ & $\mathrm{ND}$ & $>56$ \\
Family of patient 3 & & & & \\
Father (Wild/Q287X) & $\mathrm{B}$ & 4.8 & 7.7 & 4.4 \\
& $\mathrm{U}$ & 0.4 & 0.9 & 0.7 \\
Mother (Wild/L389P) & $\mathrm{B}$ & 4.0 & 8.1 & 6.9 \\
& $\mathrm{U}$ & 0.4 & 36.7 & 6.9 \\
\hline
\end{tabular}

B, blood glucose; $\mathrm{U}$, urinary glucose (normal range: $0.1-1.1)$ [mmol/1]; $\mathrm{ND}$, not determined.

olism (1). This disorder manifested hepatorenal glycogen accumulation, fasting hypoglycemia, postprandial hyperglycemia and hypergalactosemia, and Fanconi nephropathy $(2,3)$. As with our patient 3 , some patients were diagnosed with FBS as a result of neonatal screening for galactosemia $(2,11)$. Therapy initially was limited to replacement of electrolytes and vitamin D lost because of renal tubulopathy $(2,3)$. More recently, the administration of uncooked cornstarch has been reported to be beneficial for promoting growth $(8,12)$.

Santer et al. reported three mutations in the GLUT2 gene (alternative designation SLC2A2) in three families with FBS (4), and two other mutations were reported while this article was being prepared $(13,14)$. GLUT2 is one of a number of 
facilitative glucose transporters that transport glucose and other hexoses such as galactose according to the concentration gradient (15). These transporter proteins have been proposed to exist in oligomeric forms $(16,17)$. GLUT2 is present in the plasma membranes of pancreatic $\beta$ cells, hepatocytes, and intestinal and renal absorptive epithelial cells $(5,6)$. Santer et al. noted that a functional loss of GLUT2 was compatible with the clinical symptoms observed in FBS (3). In addition to being hyperglycemic and relatively hypoinsulinemic, knockout mice lacking a functioning GLUT2 gene were found to have elevated plasma concentrations of glucagon, FFA, and $\beta$-hydroxybutyrate, as well as marked glucosuria (7).

This study reports four novel mutations in three Japanese patients with FBS. Two of them, the IVS2 $-2 \mathrm{~A}>\mathrm{G}$ and Q287X mutations, result in premature translational terminations that would produce truncated proteins. Oka et al. demonstrated that a deletion mutant of GLUT1 lacking most of the carboxyterminal intracellular domain no longer could alternate in protein conformation, rendering GLUT1 functionally inactive (18). L389P and V423E were missense mutations that were not found in 100 alleles examined in healthy volunteers. Leu389 in transmembrane domain 9 is conserved in human GLUT1, 3, 4, and 5, and in GLUT2 of mouse, rat, and chicken (15). Val423, between transmembrane domains 10 and 11, is also conserved in all glucose transporters except GLUT5, which has Ile at the corresponding position. These data suggest that L389P and V423E are not common polymorphisms, but disease-causing mutations.

In the previous reports, none of the heterozygotes with mutant GLUT2 showed renal glucosuria $(3,4,19,20)$. In this study, however, OGTT findings confirmed glucosuria in the brother and the parents of patient 1, all of whom were heterozygous for the V423E mutation, and the mother of patient 3 , who was heterozygous for the L389P mutation. These data suggest the possibility that some GLUT2 mutations may cause the dominant form of familial renal glucosuria. If mutant GLUT2 proteins have a dominant-negative effect, an oligomer composed of mutant and wild-type proteins could result in abolition of glucose transport activity, as in the case with the KVLQT1 potassium channel in inherited long QT syndrome (21). Urine samples of the father of patient 3, a heterozygote of the Q287X mutation, were negative for glucose on OGTT. We speculate that heterozygosity for a nonsense mutation may not lead to glucosuria because of selective and efficient degradation of the nonsense mRNA (22). An expression study of mutant GLUT2 using Xenopus oocytes should be performed to test our hypothesis.
Acknowledgment. The authors thank Ms A. Fujimoto (Osaka City Environment and Public Health Association) for measuring galactose and other metabolites in dried blood spots.

\section{REFERENCES}

1. Fanconi G, Bickel H 1949 Die chronische Aminoacidurie (Aminosaeurediabetes oder nephrotisch-glukosurischer Zwergwuchs) bei der Glykogenose und der Cystinkrankheit. Helv Paediat Acta 4:359-396

2. Manz F, Bickel H, Brodehl J, Feist D, Gellissen K, Gescholl-Bauer B, Gilli G, Harms E, Helwig H, Nutzenadel W, Waldherr R 1987 Fanconi-Bickel syndrome. Pediat Nephrol 1:509-518

3. Santer R, Schneppenheim R, Suter D, Schaub J, Steinmann B 1998 Fanconi-Bickel syndrome - the original patient and his natural history, historical steps leading to the primary defect, and a review of the literature. Eur J Pediat 157:783-797

4. Santer R, Schneppenheim R, Dombrowski A, Gotze H, Steinmann B, Schaub J 1997 Mutations in GLUT2, the gene for the liver-type glucose transporter, in patients with Fanconi-Bickel syndrome. Nat Genet 17:324-326

5. Fukumoto H, Seino S, Imura H, Seino Y, Eddy RL, Fukushima Y, Byers MG, Shows TB, Bell GI 1988 Sequence, tissue distribution, and chromosomal localization of mRNA encoding a human glucose transporter-like protein. Proc Natl Acad Sci U S A 85:5434-5438

6. Permutt MA, Koranyi L, Keller K, Lacy PE, Scharp DW, Mueckler M 1989 Cloning and functional expression of a human pancreatic islet glucose-transporter cDNA. Proc Natl Acad Sci U S A 86:8688-8692

7. Guillam MT, Hummler E, Schaerer E, Wu JY, Birnbaum MJ, Beermann F, Schmidt A, Deriaz N, Thorens B 1997 Early diabetes and abnormal postnatal pancreatic islet development in mice lacking Glut-2. Nat Genet 17:327-330

8. Ogawa E, Fujiwara I, Abukawa D, Aikawa J, Iinuma I 1998 Achievement of normal adult height after long-term use of uncooked cornstarch in a patient with FanconiBickel syndrome. Clin Pediat Endocrinol 7(suppl 11):117-119

9. Fujimoto A 1983 Neonatal Screening. Excepta Medica, Amsterdam, pp 254-255

10. Takeda J, Kayano T, Fukomoto H, Bell GI 1993 Organization of the human GLUT2 (pancreatic $\beta$ cell and hepatocyte) glucose transporter gene. Diabetes 42:773-777

11. Muller D, Santer R, Krawinkel M, Christiansen B, Schaub J 1997 Fanconi-Bickel syndrome presenting in neonatal screening for galactosaemia. J Inherit Metab Dis 20:607-608

12. Lee PJ, Van't Hoff WG, Leonard JV 1995 Catch-up growth in Fanconi-Bickel syndrome with uncooked cornstarch. J Inherit Metab Dis 18:153-156

13. Burwinkel B, Sajad SA, Al-Sabban E, AL-Abbad A, Kilimann MW 1999 A mutation in GLUT2, not in phosphorylase kinase subunits, in hepato-renal glycogenosis with Fanconi syndrome and low phosphorylase kinase activity. Hum Genet 105:240-243

14. Akagi M, Inui K, Nakajima S, Shima M, Nishigaki T, Muramatsu T, Kokubo C, Tsukamoto H, Sakai N, Okada S 2000 Mutation analysis of two Japanese patients with Fanconi-Bickel syndrome. J Hum Genet 45:60-62

15. Mueckler M 1994 Facilitative glucose transporters. Eur J Biochem 219:713-725

16. Hebert DN, Carruthers A 1992 Glucose transporter oligomeric structure determines transporter function. Reversible redox-dependent interconversions of tetrameric and dimeric GLUT1. J Biol Chem 267:23829-23838

17. Pessino A, Hebert DN, Woon CW, Harrison SA, Clancy BM, Buxton JM, Carruthers A, Czech MP 1991 Evidence that functional erythrocyte-type glucose transporters are oligomers. J Biol Chem 266:20213-20217

18. Oka Y, Asano T, Shibasaki Y, Lin JL, Tsukuda K, Katagiri H, Akanuma Y, Takaku F 1990 C-terminal truncated glucose transporter is locked into an inward-facing form without transport activity. Nature 345:550-553

19. Tanizawa Y, Riggs AC, Chiu KC, Janssen RC, Bell DSH, Go RPC, Roseman JM, Acton RT, Permutt MA 1994 Variability of the pancreatic islet beta cell/liver (GLUT 2) glucose transporter gene in NIDDM patients. Diabetologia 37:420-427

20. Mueckler M, Kruse M, Strube M, Riggs AC, Chiu KC, Permutt MA 1994 A mutation in the Glut2 glucose transporter gene of a diabetic patient abolishes transport activity. J Biol Chem 269:17765-17767

21. Wolnik B, Schroeder BC, Kubisch C, Esperer HD, Wieacker P, Jentsch TJ 1997 Pathophysiological mechanisms of dominant and recessive KVLQT1 K channel mutations found in inherited cardiac arrhythmias. Hum Mol Genet 6:1943-1949

22. Ogasawara M, Matsubara Y, Mikami H, Narisawa K 1994 Identification of two novel mutations in the methylmalonyl-CoA mutase gene with decreased levels of mutant mRNA in methylmalonic acidemia. Hum Mol Genet 3:867-872 